\title{
Analysis of Implicitly Defined Systems
}

\author{
Fernando Paganini * John Doyle ${ }^{\dagger}$
}

\begin{abstract}
An alternative paradigm is considered for robustness analysis, where systems are described in implicit form. The central role in this formulation is played by equations rather than input-output maps. The framework for robust stability analysis is appropriately extended, and a necessary and sufficient condition is proved for the case of arbitrary structured norm bounded perturbations. Finally, the constant matrix version of this framework is considered, leading to an extension of the structured singular value $\mu$; the corresponding upper bound theory is developed fully,
\end{abstract}

\section{Introduction}

This paper is the natural continuation of previous work [13] in an extended framework for robustness analysis. This framework combines implicit characterizations of systems which appear originally in the "behavioral" approach [16] to system theory, with Linear Fractional Transformation (LFT) descriptions of uncertainty as in standard robust control. It was shown in [13] that this framework allows for the formulation of a richer class of robustness analysis problems, where an uncertain system and a finite number of Integral Quadratic Constraints (IQCs) [10] can be represented simultaneously. Tools developed in [13] for analysis of these representations have provided, in particular, for Robust $\mathcal{H}_{2}$ performance analysis. Further evidence of the potential impact of the implicit formulation is provided in [7], where a general model validation/system identification (MV/ID) problem is formulated in this fashion.

In this paper we develop this formulation more extensively from a theoretical point of view. In this respect, in Section 2 we consider general implicit systems, not restricted to the "state-space" type considered in [13]. These systems are defined in terms of equations; it is shown how robust performance problems can be cast in terms of "finding solutions to equations".

In Section 3 we review the issue of stability in this formulation; in this paper only an $l_{2}$-based theory is developed, where signals are considered a priori over $l_{2}$, but a more complete theory of stability follows similar lines [12].

Section 4 contains the main result, which is a necessary and sufficient condition for robust stability of these implicit descriptions, when the uncertainty is allowed to vary in the class of arbitrary norm bounded operators. This condition is a convex feasibility test on a constant scaling, which extends the scaled small-gain conditions for robust stability, and recent results $[14,10]$ on the necessity of these conditions for the standard input-output setting. The extension also includes $\delta I$ operator blocks in the uncertainty description.

In Section 5 we address the constant matrix version of the implicit framework. As is well known, a number of standard robust performance problems can be expressed in constant matrix form in terms of the Structured Singular Value

*Electrical Engineering, M/S 116-81, California Institute of Technology, Pasadena, CA 91125

'Control and Dynamical Systems, California Institute of Technology $\mu[6,11]$. For the implicit extension (which is essential, for example, to capture the MV/ID problem [7]) we introduce the natural generalization of $\mu$, and analyze how some of the standard theory extends, with special attention to the relationship of $\mu$ and its upper bound.

\section{The Implicit Framework for Analysis}

\subsection{Implicitly Defined Systems}

This paper deals with implicit characterizations of systems. Loosely speaking, this means that the laws governing the system and the constraints imposed on a problem under consideration are all expressed as equations imposed on a specified set of variables. A formal definition follows.

Definition 1 An implicit system is defined by two vector spaces, the variable space $W$, and the equation space $E$, and an equation operator $G: W \rightarrow E$. The behavior of the implicit system is the set $B=\operatorname{Ker}(G)=\{w \in W: G w=0\}$. The system is called linear if $G$ is a linear map.

The definition above is closely related to the so-called behavioral approach to system theory, introduced by Willems [16] In this type of formulation, all variables in a system are a priori in an equal footing, without a distinction between inputs and outputs. These descriptions arise naturally when modeling physical systems from first principles, where physical laws such as mass and energy balances are more naturally thought of as implicit equations between variables than as "signal-flow" maps. Interconnections of subsystems are reduced to superimposing equations. For a discussion of the features of this modeling paradigm, see $[16,3]$.

In our case the equations defined by $G$ play a central role, not captured entirely by the behavior, since as we shall see equation error will be added for the analysis. For the rest of this paper, all systems will be linear.

An important special case is the class of dynamicalimplicit systems, where the sets $W$ and $E$ are signal spaces. For concreteness we will consider discrete-time signals (indexed in $\mathbb{Z}$ or $\mathbb{Z}+$ ) and vector valued in $\mathbb{R}^{q}$ or $\mathbb{C}^{q}$. (i.e. $W \subset\left(\mathbb{F}^{q}\right)^{\mathbb{Z}}$, where $\mathbb{F}$ is $\mathbb{R}$ or $\mathbb{C}$. Similarly $E \subset\left(\mathbb{F}^{p}\right)^{\mathbb{Z}}$.

The choice of the signal spaces $W, E$ has strong influence in the conclusions resulting from the analysis. For instance, in most of this paper we will assume $a$ priori that the signal spaces are $l_{2}$-spaces (square summable vector sequences, $W=$ $l_{2}\left(\mathbb{C}^{q}\right), E=l_{2}\left(\mathbb{C}^{p}\right)$ ), and the conclusions are restricted to this class. In some cases in standard stability theory it must be specifically ensured that no signals outside $l_{2}$ can occur; in this case the chosen sets $W, E$ must contain a priori arbitrary sequences indexed in $\mathbb{Z}+$ (sometimes termed $l_{2}^{e}$ ); for the map $G$ to be well defined in such a class it is usually assumed to be causal, i.e. $P_{t} G P_{t}=P_{t} G \forall t$, where $P_{t}$ is the truncation operator (see [14]). We will remark briefly on this issue in Section 3. 


\subsection{Uncertainty and LFTs}

We now incorporate into the implicit paradigm deterministic descriptions of uncertainty in the style of robust control. The map $G$ is replaced by a parameterized map $G(\Delta)$, where $\Delta$ is an uncertainty operator; in this paper, we will consider uncertainty which has "spatial" structure of the form

$$
\Delta=\operatorname{diag}\left[\delta_{1} I_{r_{1}}, \ldots, \delta_{L} I_{r_{L}}, \Delta_{L+1}, \ldots, \Delta_{L+F}\right]
$$

The blocks in $\Delta$ can be used to describe real parameters, or dynamic (linear time invariant (LTI), linear time varying (LTV) or nonlinear) perturbations. In each case, there is a restricted class $\Delta$ of allowed perturbations.

In this paper we restrict ourselves to linear uncertainty blocks in discrete time. We will denote by $\mathcal{L}\left(l_{2}^{m}\right)$ the set of linear, bounded and possibly non-causal operators in $l_{2}\left(\mathbb{C}^{m}\right)$. The most general class of perturbations considered here is $\delta_{i} \in \mathcal{L}\left(l_{2}^{1}\right), \Delta_{L+j} \in \mathcal{L}\left(l_{2}^{m_{j}}\right), 1 \leq i \leq L, 1 \leq j \leq F$. All the results in this paper extend with minor changes to the continuous time case.

In the sequel, the parameterization $G(\Delta)$ for our implicit uncertain systems will be a linear fractional transformation (LFT) on the uncertainty $\Delta$, as depicted in Figure 1. For introductory material on these representations, see [4].

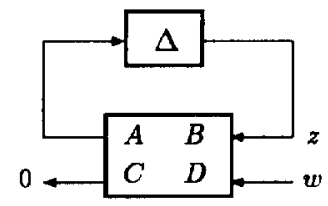

Figure 1: Implicit LFT system

In Figure 1, $\Delta$ has the structure (1), and we will assume $M=\left[\begin{array}{ll}A & B \\ C & D\end{array}\right]$ is a linear, bounded operator in $l_{2}$; an important special case is when $M$ is time-invariant.

From an implicit point of view the system in Figure 1 is characterized by the equations

$$
\varphi(\Delta, M)\left[\begin{array}{c}
z \\
w
\end{array}\right]=0, \quad \varphi(\Delta, M) \triangleq\left[\begin{array}{cc}
I-\Delta A & -\Delta B \\
C & D
\end{array}\right]
$$

A standard input-output LFT uncertain system can be easily converted to this implicit form.

\subsection{Integral Quadratic Constraints}

As was noted in [13], the implicit formulation over $l_{2}$ permits the representation of Integral Quadratic Constraints (IQCs [10]) on signals; these are time-invariant quadratic forms in signal space, of the form

$$
\int_{-\pi}^{\pi} z\left(e^{j \omega}\right) * \Pi\left(e^{j \omega}\right) z\left(e^{j \omega}\right) d \omega \leq 0
$$

where $z\left(e^{j \omega}\right)$ is the Fourier transform of an $l_{2}$ signal, II $=\mathrm{II}^{*}$ is a matrix function, assumed bounded on the unit circle. This implies that $P$ can be chosen (e.g. $P=k I$ ) such that $P^{*} P-\Pi\left(e^{j \omega}\right)>0 \forall \omega \in[0,2 \pi]$. A spectral factorization gives $P^{*} P-\Pi\left(e^{j \omega}\right)=Q^{*} Q$, (if desired, $Q$ can be chosen in $\mathcal{H}_{\infty}$ ). So $\Pi=P^{*} P-Q^{*} Q$, which reduces (3) to $\|P z\|_{2}^{2} \leq\|Q z\|_{2}^{2}$.

We now introduce the following lemmas (see the Appendix):

Lemma 1 Let $z, v \in l_{2}^{d}$. The following are equivalent:

$$
\begin{aligned}
& \text { (i) }\|v\|_{2}^{2}-\|z\|_{2}^{2} \geq 0 \\
& \text { (ii) } \exists \Delta \in \mathcal{L}\left(l_{2}^{d}\right),\|\Delta\| \leq 1, \Delta v=z
\end{aligned}
$$

Lemma 2 Let $z, v \in l_{2}^{d}$. The following are equivalent:

$$
\begin{aligned}
& \text { (i) } \int_{-\pi}^{\pi} v\left(e^{j \omega}\right) v\left(e^{j \omega}\right)^{*}-z\left(e^{j \omega}\right) z\left(e^{j \omega}\right)^{*} d \omega \geq 0 \\
& \text { (ii) } \forall \eta \in \mathbf{C}^{d},\left\|\eta^{*} v\right\|_{2} \geq\left\|\eta^{*} z\right\|_{2} \\
& \text { (iii) } \exists \delta \in \mathcal{L}\left(l_{2}\right),\|\delta\| \leq 1, \delta I_{d} v=z
\end{aligned}
$$

From the above discussion and Lemma 1 , for each $z$ satisfying (3) there exists an operator $\Delta_{C},\left\|\Delta_{c}\right\| \leq 1$ such that $P z=\Delta_{C} Q z$. So the set of $z \in l_{2}$ satisfying (3) can be described as

\section{Remarks:}

$$
\bigcup \operatorname{Ker}\left(P-\Delta_{C} Q\right)
$$

- Although for each $\Delta_{C}$ the set $\operatorname{Ker}\left(P-\Delta_{C} Q\right)$ is a linear subspace, the union of the parameterized behaviors describes a more complicated set given by (3).

- The set $\left\|\Delta_{c}\right\| \leq 1$ includes arbitrary time-varying noncausal operators. In this respect, implicit systems obtained from this procedure are only defined in $W=l_{2}$.

- Analogously, the set $\bigcup_{\left\|\Delta_{C}\right\| \leq 1} \operatorname{Ker}\left(P-\delta_{C} I Q\right)$ for scalar $\delta_{C}$ can be shown by means of Lemma 2 to correspond to the matrix-valued constraint

$$
\int_{-\pi}^{\pi} P z z^{*} P^{*}-Q z z^{*} Q^{*} d \omega \leq 0
$$

These constraints can be used to obtain tighter descriptions of disturbances and therefore reduce conservatism in robust performance analysis. In [13], "whiteness" constraints are imposed to analyze Robust $\mathcal{H}_{2}$ performance, which have the form (9): this introduces in a natural way "operatorvalued" $\delta I$ blocks in implicit uncertain systems.

To illustrate how implicit descriptions might be used for robust performance analysis, consider the uncertain inputoutput system of Figure 2, in which it is known that the input $u$ satisfies some restrictions in terms of IQCs as in (3). We want to determine whether there exist signals $u$ in the allowed class, and perturbations $\Delta$ such that the system gain is 1 or larger. This last requirement is captured by the extra "performance IQC" $\|u\|^{2}-\|y\|^{2} \leq 0$.

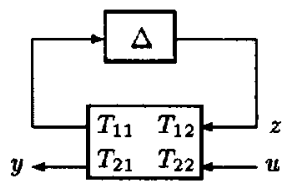

Figure 2: Input/Output LFT system

The implicit equations for the system, the IQCs on $u$ and the performance IQC are captured respectively by (10),(11) and (12), with $\Delta_{C}, \Delta_{P}$ arbitrary norm bounded operators.

$$
\begin{aligned}
& {\left[\begin{array}{ccc}
I-\Delta T_{11} & 0 & -\Delta T_{12} \\
T_{21} & -I & T_{22}
\end{array}\right]\left[\begin{array}{l}
z \\
y \\
u
\end{array}\right]=0} \\
& \left(P-\Delta_{C} Q\right) u=0 \\
& {\left[\begin{array}{ll}
\Delta_{P} & I
\end{array}\right]\left[\begin{array}{l}
y \\
u
\end{array}\right]=0}
\end{aligned}
$$

The superposition of (10), (11) and (12) gives an implicit description for the robust performance analysis problem, which essentially reduces to the question: "Does there exist a perturbation $\left(\Delta, \Delta_{C}, \Delta_{P}\right)$ with non-trivial $l_{2}$ kernel?" This type of questions are addressed in the rest of this paper. 


\section{Stability in Implicit Systems}

The concept of stability in standard system theory has been given two interpretations.

In the first place, from the point of view of dynamical systems, stability ensures that solutions do not exist where the signals are unbounded (e.g. escape $\boldsymbol{l}_{2}$ ). This notion is directly tied to causal systems (where the operations on unbounded signals are well defined).

Secondly, from an operator theoretic point of view, stability is associated with the fact that small errors or disturbances do not get arbitrarily amplified by a system interconnection. This notion can be stated for non-causal systems, but it must be assumed a priori that the signals are in a space (e.g. $l_{2}$ ) where the operators are well defined.

Although these two versions can be proved equivalent in many special cases of causal systems, we will find it useful to distinguish the two for the extension to the implicit framework, since we are led naturally to include non-causal perturbations as explained in Section 2.3. We will term the first notion "stability" and the second " $l_{2}$-stability" (the notion could also be defined in other signal spaces). This paper deals with $l_{2}$-stability, the stability case is referred to [12]

\section{1 $l_{2}$ Stability and Robust Stability}

Definition 2 Let $G w=0$ denote an implicit linear system over $l_{2}$ (i.e., $w \in l_{2}^{q}, G: l_{2}^{q} \rightarrow l_{2}^{p}$ ). The system is $l_{2}$-stable if $\inf \{\|G w\|:\|w\|=1\}>0$.

According to this definition, $l_{2}$-stability implies that the $l_{2}$ behavior $B$ of the system is the trivial space, and that this property is not "sensitive" to equation error. More precisely, if $G w=e$, with $\|w\|=1$, then the equation error $e$ is bounded below in norm by a positive number. Equivalently, by the Open Mapping Theorem, $l_{2}$-stability states that $G$ has a left inverse $L: l_{2}^{p} \rightarrow l_{2}^{q}, L G=I$, and $L$ is a bounded operator. We will now compare this definition with the standard one, by considering the feedback interconnection of Figure 3 . The maps $M, N$ are (not necessarily causal) operators on $l_{2}$.

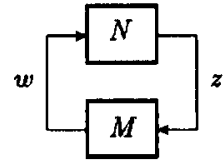

Figure 3: Standard feedback interconnection

This interconnection can be represented by the following equations in the variables $w, z$ :

$$
\left[\begin{array}{cc}
I & -M \\
-N & I
\end{array}\right]\left[\begin{array}{l}
w \\
z
\end{array}\right]=0
$$

Definition 2 implies that arbitrarily small equation errors in (13) (which correspond to interconnection errors in Figure 3) do not allow $\left[w^{\prime}, z^{\prime}\right]^{\prime}$ of norm 1 , which corresponds to the standard notion of stability in the operator theoretic sense. The only difference is that we are not requiring the operator $G=\left[\begin{array}{cc}I & -M \\ -N & I\end{array}\right]$ to be invertible, only left invertible. In other words, $G$ need not be onto $l_{2}^{p}$ in our definition: the equation errors need not be free to vary over $l_{2}^{p}$. The reason for this weakened definition in the case of implicit systems is that we want to extend the notion to systems which are over-constrained (more equations than variables), such as the example considered in (10-12). In these cases, the operator will not be onto in general, and this should not be required: the equation errors need not be "free" since they are not physical noises (which should be included in any model); they just provide a means of testing sensitivity of implicit equations.
For the case of uncertain systems, robust stability means as usual stability for each element in the class. We give the following definition for LFT systems:

Definition 3 The implicit system (2) has robust $l_{2}$-stability if it is $l_{2}$ stable $\forall \Delta \in \mathbf{B}_{\mathbf{\Delta}}$. Robust $l_{2}$-stability is uniform if

$$
\inf \left\{\left\|\varphi(\Delta, M)\left[\begin{array}{c}
z \\
w
\end{array}\right]\right\|: \Delta \in \mathbf{B}_{\Delta},\left\|\begin{array}{c}
z \\
w
\end{array}\right\|=1\right\}>0
$$

Robust $l_{2}$ stability implies that for each $\Delta \in \mathbf{B}_{\Delta}, \varphi(\Delta, M)$ has a bounded left inverse; uniform robust $l_{2}$ stability means that there is a uniform bound on the norms of these left inverses across $\mathbf{B}_{\boldsymbol{\Delta}}$; this extends standard definitions [14]. The notion of stability is "internal" in the sense that the variable $z$ is included in the variable space $W$.

The input-output robust performance problem posed in Section 2.3 converts exactly to robust $l_{2}$-stability of the corresponding implicit system. This motivates the main result of this paper, given in section 4, which provides necessary and sufficient conditions for robust $l_{2}$-stability in the implicit case. We first state the following proposition which simplifies the representation:

Proposition 1 The implicit system (2) has (uniform) robust $l_{2}$-stability if and only if

(i) $D$ is $l_{2}-$ stable, with bounded left inverse $L$.

(ii) The implicit system $\left[\begin{array}{c}I-\Delta \hat{A} \\ \hat{C}\end{array}\right] z=0$ has (uniform) robust $l_{2}$-stability, where $\hat{A}=A-B L C, \hat{C}=C-D L C$

Proof: (Necessity) If the system (2) is robust $l_{2}$-stable, setting $\Delta=0, \varphi(0, M)=\left[\begin{array}{ll}I & 0 \\ C & D\end{array}\right]$ has bounded left inverse, therefore (i) holds. Fix $\Delta \in \mathbf{B}_{\mathbf{\Delta}}$; since

$$
\left[\begin{array}{cc}
I & \Delta B L \\
0 & I
\end{array}\right] \varphi(\Delta, M)\left[\begin{array}{cc}
I & 0 \\
-L C & I
\end{array}\right]=\left[\begin{array}{cc}
I-\Delta \hat{A} & 0 \\
\hat{C} & D
\end{array}\right]
$$

and $\varphi(\Delta, M)$ is left invertible, so is $\left[\begin{array}{c}I-\Delta \hat{A} \\ \hat{C}\end{array}\right]$.

(Sufflciency) If [ $\left[\begin{array}{ll}L_{11} & L_{12}\end{array}\right]$ is a bounded left inverse for $\left[\begin{array}{c}I-\Delta \hat{A} \\ \hat{C}\end{array}\right]$, then (note that by definition of $\hat{C}, L \hat{C}=0$ ),

$$
\left[\begin{array}{cc}
L_{11} & L_{12} \\
0 & L
\end{array}\right]\left[\begin{array}{cc}
I-\Delta \hat{A} & 0 \\
\hat{C} & D
\end{array}\right]=\left[\begin{array}{cc}
I & L_{12} D \\
0 & I
\end{array}\right]
$$

invertible, therefore $\left[\begin{array}{cc}I-\Delta \hat{A} & 0 \\ \hat{C} & D\end{array}\right]$ left invertible and so is $\varphi(\Delta, M)$ using (15)

Condition ( $i$ ) is a nominal stability condition, which must be satisfied for the problem to be nontrivial. We consider from now on the simplified setup of (ii) (renaming $\hat{A}, \hat{C}$ as $A, C$ ):

$$
\left[\begin{array}{c}
I-\Delta A \\
C
\end{array}\right] z=0
$$

\section{A Necessary and Sufficient Condition}

In this section we treat a special case of the robustness analysis problem (17): assume that $A, C$, are $L T I$ bounded (stable) operators on $l_{2}$ (possibly non-causal), and that the uncertainty set $\Delta$ is the class of all structured linear operators on $l_{2}$.

The main result in this paper is a necessary and sufficient condition for robust $l_{2}$-stability under these conditions. This result is an extension of recent results of Shamma [14] and 
Megretski [10] (and previously in the $l_{\infty}$ setting by Khammash [8]), regarding the necessity of a "scaled small gain" condition for robust stability in the standard input output setting, where $[8,14]$ use the LFT formulation, and $[10]$ considers the $\mathrm{QQC}$ version. All these results have considered only full block perturbations.

In what follows we will provide an extension to the implicit framework of these results. In view of the duality between IQCs and implicit LFT descriptions that was shown in Section 2.3 , the theorem given below strongly parallels the S-procedure losslessness results of Megretski and Treil [10]. The main extension that needed is to capture the $\delta I$ blocks, which are not described by scalar valued IQCs; this is done by extending the " $\nabla$ set" method in standard $\mu$-analysis [11]

We begin with some notation. For a general delta structure $\Delta$ of the form (1), let $Y$ be the set of constant, hermitian scaling matrices $\mathbf{Y}$ that commute with the elements in $\Delta$,

$$
Y \triangleq\left\{Y=\operatorname{diag}\left[Y_{1}, \ldots, Y_{L}, y_{L+1} I_{m_{1}}, \ldots, y_{L+F} I_{m_{F}}\right]\right.
$$

$$
\left.Y_{i}=Y_{i}^{*}, i=1 \ldots L, y_{L+j} \in \mathbf{R}, j=1 \ldots F\right\}
$$

$Y$ is a real yector space, and we can define an inner product:

$$
\langle Y, \bar{Y}\rangle=\sum_{i=1}^{L} \operatorname{tr}\left(Y_{i} \bar{Y}_{i}\right)+\sum_{j=1}^{F} y_{L+j} \bar{y}_{L+j}
$$

Two important subsets of $Y$ are $X=\{Y \in Y, Y>0\}$, and $\overline{\mathbf{X}}=\{Y \in Y, Y \geq 0\}$, the set of positive and nonnegative scalings, respectively. They are both convex cones in $\mathbf{Y}$.

Given a vector $z(t) \in l_{2}^{n}$, the block structure introduces a natural partition $z=\left[z_{1}^{\prime} \ldots z_{L}^{\prime} z_{L+1}^{\prime} \ldots z_{L+F}^{\prime}\right]^{\prime}$.

Given an LTI stable system $A(\lambda)$, an analogous notation is used for the partition of $A z \in l_{2}^{n}$. Consider the following quadratic functions of $z \in l_{2}^{n}$ :

$$
\begin{gathered}
\Phi_{i}(z)=\sum_{t=-\infty}^{\infty}(A z)_{i}(t)(A z)_{i}(t)^{*}-z_{i}(t) z_{i}(t)^{*} \quad i=1 \ldots L \\
\sigma_{j}(z)=\left\|(A z)_{L+j}\right\|^{2}-\left\|z_{L+j}\right\|^{2} \\
\Lambda(z)=\operatorname{diag}\left[\Phi_{1}(z), \ldots, \Phi_{L}(z), \sigma_{1}(z) I_{m_{1}}, \ldots, \sigma_{F}(z) I_{m_{F}}\right]
\end{gathered}
$$$$
\text { Now let } C \text { be LTI, stable, } \epsilon>0 \text {. Define a subset of } Y \text {, }
$$

$$
\nabla^{\epsilon}=\{\Lambda(z):\|z\|=1,\|C z\|<\epsilon\}
$$

Lemma $3 \bar{\nabla}^{\epsilon}$ (closure of $\left.\nabla^{\epsilon}\right)$ is convex and compact in $\mathbf{Y}$.

Theorem 1 Let $A, C$ be LTI stable systems, $\nabla^{\epsilon}$ as in (20). Assume $\Delta$ is the set of structured, otherwise arbitrary linear operators in $l_{2}^{n}$. The following are equivalent:

(i) (17) has uniform $l_{2}$ robust stability.

(ii) $\exists \epsilon>0$ such that $\bar{\nabla}^{e} \cap \overline{\mathrm{X}}=\emptyset$

(iii) $\exists$ constant $X \in \mathrm{X}: A^{*} X A-X-C^{*} C<0$

In (iii) above, the constraint (21) is of the form $\Psi<0$ where $\Psi$ is a self-adjoint operator on $l_{2}$; this must be interpreted as a strong version of negative definiteness, $\langle\Psi u, u\rangle \leq$ $-\rho\|u\|^{2}$ for some $\rho>0$.

Proof: Lemma 3 and Theorem 1 are proved in the Appendix.

\section{The Constant Matrix Case}

In many important cases, robustness analysis can be conducted in a constant matrix representation, which is essential if computational tests are to be derived. These have the form $\left[\begin{array}{c}I-\Delta A \\ C\end{array}\right] z=0, A \in \mathbb{C}^{n \times n} C \in \mathbb{C}^{\infty \times n} \Delta \in \Delta \subset \mathbb{C}^{n \times n}$
The structure $\boldsymbol{\Delta}$ is still of the form (1), and it could also have blocks restricted to be real as in [17]. By analogy with Definition 2, we say that the implicit system (22) is stable if

$$
\operatorname{Ker}\left[\begin{array}{c}
I-\Delta A \\
C
\end{array}\right]=0 \forall \Delta \in \mathbf{B}_{\Delta}
$$

\subsection{Motivation for Constant Matrix Problems}

One case which reduces to a problem like (23) is for state space implicit descriptions in discrete time, as considered in [13]. Assume that $M$ in Figure 1 is a finite dimensional LTI system. By writing a state space realization of this system we obtain a new implicit description, where $M$ is replaced by a constant matrix, and the delta structure $\Delta$ by an augmented structure $\Delta_{S}=\operatorname{diag}[\lambda I, \Delta]$ where $\lambda$ is the delay operator; under some conditions [13] a constant matrix test results.

Another case is for time-invariant systems, which can be reduced to constant matrix analysis plus a frequency sweep:

Proposition 2 Consider the implicit system (23), where A, $C$ are finite dimensional LTI systems and $\Delta$ is LTI. Then the system has (uniform) robust $l_{2}$ stability if and only if

$$
\operatorname{Ker}\left[\begin{array}{c}
I-\Delta_{0} A\left(e^{j \omega}\right) \\
C\left(e^{j \omega}\right)
\end{array}\right]=0 \forall \Delta_{0} \in \mathbf{B}_{\Delta 0}, \forall \omega \in[-\pi, \pi],
$$

where $A\left(e^{j \omega}\right), C\left(e^{j \omega}\right)$ are the frequency responses of $A, C$, and $\Delta_{0}$ is a constant complex perturbation with the same structure as the original $\Delta$.

Proof: Assume (24) holds. Then for any LTI $\Delta \in \mathbf{B}_{\mathbf{\Delta}}$, $\Delta\left(e^{j \omega}\right) \in \mathbf{B}_{\Delta_{0}}$ holds almost everywhere and therefore

$$
\underline{\sigma}\left[\begin{array}{c}
I-\Delta\left(e^{j \omega}\right) A\left(e^{j \omega}\right) \\
C\left(e^{j \omega}\right)
\end{array}\right] \geq \epsilon>0
$$

holds a.e. where $g$ denotes minimum singular value. From here it is easy to obtain a left inverse $L\left(e^{j \omega}\right)$ of $\left[\begin{array}{c}I-\Delta\left(e^{j \omega}\right) A\left(e^{j \omega}\right) \\ C\left(e^{j \omega}\right)\end{array}\right]$, with $\left\|L\left(e^{j \omega}\right)\right\|_{\infty}<\infty$, therefore a bounded operator in $l_{2}$. Conversely, if (24) fails for $\omega_{0}, \Delta_{0}$, with kernel given by $z_{0}$, it is easy to construct $\Delta \in \mathbf{B}_{\mathbf{\Delta}}, \mathrm{fi}-$ nite dimensional such that $\Delta\left(e^{j \omega_{0}}\right)=\Delta_{0}$. Consider now a sequence of signals $z^{(k)}$ with norm 1 , in the direction $z_{0}$, and spectrum supported in $\left[\omega_{0}-\frac{1}{k}, \omega_{0}-\frac{1}{k}\right]$. It easy to show that $\left[\begin{array}{c}I-\Delta A \\ C\end{array}\right] z^{(k)} \stackrel{k \rightarrow \infty}{\longrightarrow} 0$, violating robust stability.

Finally, another situation which yields a constant matrix representation arises from finite horizon analysis problems in the time domain [15]. An important example of this is a class of model validation/ system identification problems [7], for which the implicit extension is required.

\subsection{An Extended Structured Singular Value $\mu$}

The definition of stability (23) is a natural extension of the structured singular value $\mu[6,11]$ which corresponds to the case where the $C$ equations are not present. Inspired by this, we can give the following definition:

Definition 4 The structured singular value of the implicit system (22) is defined as

$\mu_{\Delta, C}(A) \triangleq\left(\min \left\{\bar{\sigma}(\Delta): \Delta \in \Delta, \operatorname{Ker}\left[\begin{array}{c}I-\Delta A \\ C\end{array}\right] \neq 0\right\}\right)^{-1}$

If $\operatorname{Ker}\left[\begin{array}{c}I-\Delta A \\ C\end{array}\right]=0 \forall \Delta \in \Delta$, define $\mu_{\Delta, C}(A) \triangleq 0$. 
A restatement of this definition is to say that $(22)$ is stable if and only if $\mu_{\Delta, C}(A)<1$. This raises the question of computing the function $\mu_{\Delta, C}(A)$; as in the standard case, exact computation is not easy and we must rely in upper and lower bounds.

For the lower bound side, note that the kernel condition in (26) is equivalent to $\operatorname{Ker}(B-\Delta F) \neq 0$, where $B=C_{\perp}^{*}$ $F=A C_{\perp}^{*}$, and $C_{\perp}$ is a matrix whose columns form a basis of $\operatorname{Ker}(C)$. This resembles a generalized eigenvalue problem, whereas the condition $\operatorname{Ker}(I-\Delta A) \neq 0$ of the unconstrained case parallels the regular eigenvalue problem. Algorithms which rely on this observation have been developed [11, 17] for the standard case, and presumably can be extended to the implicit case. Some initial work is documented in [7]

For the discussion of the upper bounds, it is convenient to introduce a "static" version of the $\nabla^{\epsilon}$ set defined above. For $\zeta \in \mathbb{C}^{n}$, let

$$
\begin{aligned}
& \Phi_{i}^{0}(\zeta)=(A \zeta)_{i}(A \zeta)_{i}^{*}-\zeta_{i} \zeta_{i}^{*} \quad i=1 \ldots L \\
& \sigma_{j}^{0}(\zeta)=(A \zeta)_{L+j}^{*}(A \zeta)_{L+j}-\zeta_{L+j}^{*} \zeta_{L+j} \quad j=1 \ldots F \\
& \Lambda^{0}(\zeta)=\operatorname{diag}\left[\Phi_{1}^{0}(\zeta), \ldots, \Phi_{L}^{0}(\zeta), \sigma_{1}^{0}(\zeta) I_{m_{1}}, \ldots, \sigma_{F}^{0}(\zeta) I_{m_{F}}\right]
\end{aligned}
$$

Define $\nabla^{0}=\left\{\Lambda^{0}(\zeta): C \zeta=0,|\zeta|=1\right\} \subset \mathbf{Y}$. Constant matrix versions of Lemmas 1 and 2 show that $\mu_{\Delta, C}(A)<1$ if and only if $\nabla^{0} \cap \bar{X}=\phi$.

Now consider the following Linear Matrix Inequalities (LMIs), which can be easily shown to be equivalent:

$$
\begin{aligned}
& \exists X \in \mathbf{X}: A^{*} X A-\beta^{2} X-C^{*} C<0 \\
& \exists X \in \mathbf{X}: C_{\perp}\left(A^{*} X A-\beta^{2} X\right) C_{\perp}^{*}<0
\end{aligned}
$$

Testing whether an LMI is satisfied is a convex feasibility problem, for which interior point methods are available (see [2]). Define

$$
\hat{\mu}_{\Delta, C}(A)=\inf \{\beta>0:(27) \text { is satisfied }\}
$$

By the same methods as in Theorem 1 we can show that $\hat{\mu}_{\Delta, C}(A)<1$ if and only if $\operatorname{co}\left(\nabla_{0}\right) \cap \overline{\mathbf{X}}=\phi$, where $\operatorname{co}\left(\nabla_{0}\right)$ is the convex hull of $\nabla_{0}$. A consequence of this is $\mu_{\Delta, C}(A) \leq$ $\hat{\mu}_{\Delta, C}(A),(\hat{\mu}$ is an upper bound for $\mu$ ).

In this static case, the upper bound will be strict in general; equivalently, LMI (27) with $\beta=1$ is not a necessary test for stability; the step that fails in extending Theorem 1 is that the convexity of $\nabla^{\circ}$ can no longer be guaranteed (so $\nabla^{0} \cap \bar{X}=\phi$ does not imply $\left.\operatorname{co}\left(\nabla^{0}\right) \cap \overline{\mathrm{X}}=\phi\right)$.

In special cases, the equality of $\mu, \hat{\mu}$ holds; we will call a structure $\Delta \mu$-simple in the implicit case if $\mu_{\Delta, C}(A)=$ $\hat{\mu}_{\Delta, C}(A)$ for any matrices $A, C$. We have the following

Proposition 3 The following structures are $\mu$-simple in the implicit case:

(i) $\Delta=\{\delta I: \delta \in \mathbb{C}\} \subset \mathbb{C}^{n \times n}$;

(ii) $\Delta=C^{n \times n}$;

(iii) $\Delta=\left\{\operatorname{diag}\left[\Delta_{1}, \Delta_{2}\right]: \Delta_{i} \in \mathbb{C}^{m_{i} \times m_{i}}\right\} \subset \mathbb{C}^{n \times n}$.

(iv) $\Delta=\left\{\right.$ diag $\left.\left[\Delta_{1}, \Delta_{2}\right]: \Delta_{i} \in \mathbb{R}^{m_{i} \times m_{i}}\right\} \subset \mathbb{R}^{n \times n}$, where $A, C$ are real.

\section{Remarks on the Proof:}

For case (i), the definition of stability (23) is equivalent by the PBH test, to the condition $(C, A)$ detectable in the usual sense. It is well known that in this case the condition can be captured by an LMI such as (27). In fact, extension of these PBH-type tests to structured uncertainty for input-output systems are further exploited in [9].

For cases (ii), (iii), it can be shown that the set $\nabla^{0}$ is in fact convex. This follows from [6], where the range space of two complex hermitian forms is considered. Case (iv) requires a refinement of the methods in [6], and is deferred to [12].
In standard $\mu[11]$, two additional complex structures are $\mu$-simple (diag $\left[\delta_{1} I, \Delta_{2}\right]$ and $\left.\operatorname{diag}\left[\Delta_{1}, \Delta_{2}, \Delta_{3}\right]\right)$ This is no longer true in the implicit formulation. We now show a counterexample for the first case.

Example

Let $\Delta=\operatorname{diag}\left[\delta I_{2}, \Delta_{0}\right], \Delta_{0} \in \mathbb{C}^{2 \times 2}$

$$
B=C_{\perp}^{*}=\left[\begin{array}{ll}
1 & 0 \\
0 & 1 \\
4 & 0 \\
0 & 4
\end{array}\right] ; \quad F=A C_{\perp}^{*}=\left[\begin{array}{ll}
2 & 0 \\
0 & 3 \\
3 & 2 \\
2 & 2
\end{array}\right]
$$

The top half of $B-\Delta F$ is $\left[\begin{array}{cc}1-2 \delta & 0 \\ 0 & 1-3 \delta\end{array}\right]$, so the kernel is nontrivial only for $\delta=1 / 2, \delta=1 / 3$. In the first case, the kernel must be the span of $[1,0]^{\prime}$ therefore $\left[\begin{array}{l}4 \\ 0\end{array}\right]=\Delta_{0}\left[\begin{array}{l}3 \\ 2\end{array}\right]$. This implies $\bar{\sigma}\left(\Delta_{0}\right) \geq 4 / \sqrt{13}$. A similar argument with $\delta=$ $1 / 3$, shows that for a nontrivial kernel, $\bar{\sigma}\left(\Delta_{0}\right) \geq \sqrt{2}$. The first perturbation is smaller and therefore $\mu_{\Delta, C}(A)=\sqrt{13} / 4<1$.

For $\hat{\mu}$, consider LMI (28), $\beta=1$, and write $X=$ $\operatorname{diag}\left[X_{0}, I_{2}\right]$, with $X_{0}=\left[\begin{array}{cc}x & y \\ y^{*} & z\end{array}\right]$. Some algebra gives

$$
C_{\perp}\left(A^{*} X A-X\right) C_{\perp}^{*}=\left[\begin{array}{cc}
3(x-1) & 5(y+2) \\
5\left(y^{*}+2\right) & 8(z-1)
\end{array}\right]
$$

For (30) to be negative definite, and $X>0$, we must have

$0<x<1 ; 0<z<1 ;|y|^{2}<x z ;|y+2|^{2}<\frac{24}{25}(1-x)(1-z)$

This implies $|y|<1,|y+2|<1$ which is impossible, so there is no solution to the LMI (28). Therefore $\hat{\mu}_{\Delta, C}(A) \geq 1$.

\section{Remark:}

If we consider $A, C$ constant matrices but $\Delta$ is allowed to vary in the class of arbitrary $l_{2}$ operators, Theorem 1 implies that $\hat{\mu}_{\Delta, C}(A)<1$ is the exact $l_{2}$-stability test. This amounts to an infinite augmentation of the implicit $\mu$ problem. In fact, since the $\nabla$-sets are in a finite dimensional space $Y$, it can be shown that a finite augmentation of the implicit $\mu$-problem will have an exact upper bound. An augmentation of size $d+1$ suffices, where $d$ is the dimension of $Y$, which can be shown (see [12]) by using the fact that the convex hull in $Y$ is generated by convex combinations of at most $d+1$ elements in the set. A similar result (for standard $\mu$-analysis) has been obtained using very different methods in [1].

In comparison, if $A, C$ have unbounded memory, an infinite horizon augmentation (as in Section 4) is required.

\section{Conclusions}

The work reported in this paper, together with [13], pro vides the foundation for a more general robustness analysis theory, which extends the standard theory based on the small gain theorem. In this approach, we abandon the concepts of "input-output maps" and "gains" in favor of equations and signal constraints, and the central analysis question is to test whether there exist solutions to these equations.

It is clear by the results of this paper that nothing is lost, from a mathematical point of view, by adopting this approach for analysis instead of the standard input-output formulation; on the contrary, $[13,7]$ show evidence of substantial advantages. Moreover, there is evidence [5] of further advantages for design.

There are still reasons to preserve the standard "signalflow" approach, which has led to a large body of knowledge, since some of its intuitive value might be lost in the "equations" approach.

The conclusion is, however, that if research is not confined to the traditional paradigm the potential of the resulting the ory will be greatly enhanced. 


\section{Appendix}

\section{Proof of Lemmas 1 and 2}

The only non-trivial implication is (ii) $\Rightarrow$ (iii) in Lemma 2 .

If $\hat{v}_{1}, \ldots \hat{v}_{r}$, is an orthonormal basis of the subspace of $l_{2}$ spanned by the coordinates $v_{1}, \ldots v_{d}$ of $v$, we write $\hat{v}=P v$, where $P$ is an invertible matrix, $\hat{v}=\left[\hat{v}_{1}, \ldots \hat{v}_{r}, 0, \ldots 0\right]^{\prime}$. Let $\hat{z}=P z$, then (ii) implies $\hat{z}=\left[\hat{z}_{1}, \ldots \hat{z}_{r}, 0, \ldots .0\right]^{\prime}$, and

$\left\|\eta_{1} \hat{v}_{1}+\ldots+\eta_{r} \hat{v}_{r}\right\| \geq\left\|\eta_{1} \hat{z}_{1}+\ldots+\eta_{r} \hat{z}_{r}\right\| \forall \eta_{1}, \ldots \eta_{r} \in \mathbb{C}$

Now define $\delta: \xi \mapsto \sum_{i=1}^{r}\left\langle\xi, \hat{v}_{i}\right\rangle \hat{z}_{i} ;$ then $\delta: \hat{v}_{i} \mapsto \hat{z}_{i}, i=1 \ldots r$, so $\delta I \hat{v}=\hat{z}$ which implies $\delta I v=z$. Also, by (32) and the Bessel inequality, $\|\delta \xi\| \leq\left\|\sum_{i=1}^{r}\left\langle\xi, \hat{v}_{i}\right) \hat{v}_{i}\right\| \leq\|\xi\|$. So $\|\delta\| \leq 1$.

\section{Proof of Lemma 3}

The stability of $A$ implies that $\nabla^{\epsilon}$ is bounded, therefore $\nabla^{\epsilon}$ is compact. For the convexity proof, it suffices to show that $\operatorname{co}\left(\nabla^{\epsilon}\right) \subset \nabla^{\epsilon}$, where $\operatorname{co}\left(\nabla^{\epsilon}\right)$ is the convex hull of $\nabla^{\epsilon}$. Consider the convex combination $\alpha \Lambda(z)+(1-\alpha) \Lambda(v)$ of two elements of $\nabla^{\epsilon} .(\|z\|=\|v\|=1,\|C z\|<\epsilon,\|C v\|<\epsilon, 0<\alpha<1)$. Define $v^{(k)}=(\alpha)^{\frac{1}{2}} z+(1-\alpha)^{\frac{1}{2}} \lambda^{k} v$. Then

$$
\begin{aligned}
& \left\|v^{(k)}\right\|^{2}=\alpha\|z\|^{2}+(1-\alpha)\|v\|^{2}+2\left(\alpha-\alpha^{2}\right)^{\frac{1}{2}}\left(z, \lambda^{k} v\right\rangle \quad(33) \\
& \left\|C v^{(k)}\right\|^{2}=\alpha\|C z\|^{2}+(1-\alpha)\|C v\|^{2}+2\left(\alpha-\alpha^{2}\right)^{\frac{1}{2}}\left(C z, \lambda^{k} C v\right)
\end{aligned}
$$

where (34) uses the time invariance of $C$. The last terms in (33), (34) have limit 0 as $k \rightarrow \infty$. Therefore $\left\|v^{(k)}\right\|^{2} \stackrel{k \rightarrow \infty}{\longrightarrow}$ $\alpha\|z\|^{2}+(1-\alpha)\|v\|^{2}=1$, and $\left\|C \frac{v^{(k)}}{\left\|v^{(k)}\right\|}\right\|<\epsilon$ for $k \geq k_{0}$. A similar argument using the time invariance of $A$, shows that

$$
\begin{gathered}
\Phi_{i}\left(v^{(k)}\right) \stackrel{k \rightarrow \infty}{\longrightarrow} \alpha \Phi_{i}(z)+(1-\alpha) \Phi_{i}(v) \quad i=1 \ldots L \\
\sigma,\left(v^{(k)}\right) \stackrel{k=\infty}{\longrightarrow} \alpha \sigma_{j}(z)+(1-\alpha) \sigma_{j}(v) \quad j=1 \ldots F
\end{gathered}
$$

This gives $\Lambda\left(\frac{v^{(k)}}{\left\|v^{(k)}\right\|}\right) \stackrel{k \rightarrow \infty}{\longrightarrow} \alpha \Lambda(z)+(1-\alpha) \Lambda(v)$, therefore $\alpha \Lambda(z)+(1-\alpha) \Lambda(v) \in \nabla^{\epsilon}$. A convex combination of any number of terms can be handled in a similar way.

\section{Proof of Theorem 1}

(i) $\Rightarrow$ (ii) By contradiction, assume that $\bar{\nabla}^{c} \cap \overline{\mathbf{X}} \neq \emptyset \epsilon>0$. For a fixed $\epsilon>0$, we can therefore find $z \in l_{2}$ such that $\|z\|=1,\|C z\|<\epsilon$, and $\Lambda(z)+\epsilon^{2} I \geq 0$. Let $\xi=A z$, we have

$$
\begin{array}{rl}
\left\|\xi_{L+j}\right\|^{2}-\left\|z_{L+j}\right\|^{2}+\epsilon^{2} \geq 0 & j=1 \ldots F \\
\sum_{t=-\infty}^{\infty} \xi_{i}(t) \xi_{i}(t)^{*}-z_{i}(t) z_{i}(t)^{*}+\epsilon^{2} I \geq 0 & i=1 \ldots L
\end{array}
$$

Focusing on (37), a slight extension of Lemma 1 shows that there exists an operator $\Delta_{L+j},\left\|\Delta_{L+j}\right\| \leq 1$ and an error signal $e_{L+j},\left\|e_{L+j}\right\|=O(\epsilon), \Delta_{L+j} \xi_{L+j}+e_{L+j}=z_{L+j}$.

A similar argument (extending Lemma 2) is used for (38). The result is a structured LTV operator $\Delta,\|\Delta\| \leq 1$ and an error signal $e,\|e\|=O(c)$ such that $(I-\Delta A) z=e$. Therefore

$$
\left\|\left[\begin{array}{c}
I-\Delta A \\
C
\end{array}\right] z\right\|=O(\epsilon) \text {. }
$$

Since $\epsilon$ is arbitrary, uniform robust $l_{2}$-stability is violated. (ii) $\Rightarrow$ (iii)

$\nabla^{e}$ and $\mathbf{X}$ are disjoint convex sets in the inner product space $\mathbf{Y}, \bar{\nabla}^{\epsilon}$ is compact and $\overline{\mathbf{X}}$ is closed. By a hyperplane separation argument we find $X \in \mathrm{Y}, \eta>0$ such that

$\langle X, \Lambda\rangle \leq \alpha-\eta\left\langle\alpha \leq\langle X, Y\rangle \forall \Lambda \in \bar{\nabla}^{\epsilon}, Y \in \overline{\mathrm{X}}\right.$

Since $\bar{X}$ is a cone, $\alpha$ can be chosen to be 0 . Now

$$
\langle X, Y\rangle \geq 0 \forall Y \in Y, Y \geq 0 \Longrightarrow X \geq 0
$$

A small perturbation of $X$ ensures $X>0(X \in X)$, and by continuity and compactness of $\bar{\nabla}^{\mathrm{c}}$ we can modify $\eta$ to achieve $\langle X, \Lambda\rangle \leq-\eta<0 \forall \Lambda \in \bar{\nabla}^{\circ}$ for the new $X$. Furthermore, by scaling down $X$ (and $\eta$ ) we can ensure (42) holds with $\left\|A^{*} X A-X\right\|=\gamma^{2}<\epsilon^{2}$. Now for any $z=l_{2},\|z\|=1$, the definition of $\Lambda$ gives

$$
\langle X, \Lambda(z)\rangle=\left\langle\left(A^{*} X A-X\right) z, z\right\rangle
$$

Let $\Psi=A^{*} X A-X-C^{*} C$.

If $\|C z\|<\epsilon$, then $\Lambda(z) \in \nabla^{\epsilon}$ so $\langle\Psi z, z\rangle \leq\left\langle\left(A^{*} X A-\right.\right.$ $X) z, z\rangle=\langle X, \Lambda(z)\rangle \leq-\eta<0$ by (42).

If $\|C z\| \geq \epsilon$, then $(\Psi z, z\rangle \leq\left\langle\left(A^{*} X A-X\right) z, z\right\rangle-\|C z\|^{2} \leq$ $\gamma^{2}-\epsilon^{2}<0$.

So $\langle\Psi z, z\rangle \leq \max \left(-\eta, \gamma^{2}-\epsilon^{2}\right)$ for $\|z\|=1, \Longrightarrow \Psi<0$.

(iii) $\Rightarrow$ (i)

Fix $X>0$ which solves (21). The notion of negative definiteness allows by continuity to find $\alpha<1$ such that $A^{*} X A-\alpha X-C^{*} C<0$. Let $\Delta,\|\Delta\| \leq 1, e, z$ satisfy

Then $\quad\left[\begin{array}{c}I-\Delta A \\ C\end{array}\right] z=e=\left[\begin{array}{l}e_{1} \\ \epsilon_{2}\end{array}\right]$

$\left\|\Delta X^{\frac{1}{2}} A z\right\|^{2} \leq\left\|X^{\frac{1}{2}} A z\right\|^{2}=\left\langle A^{*} X A z, z\right\rangle \leq \alpha\left\|X^{\frac{1}{2}} z\right\|^{2}+\left\|e_{2}\right\|^{2}$

Therefore $\left\|X^{\frac{1}{2}} z-\Delta X^{\frac{1}{2}} A z\right\| \geq(1-\sqrt{\alpha})\left\|X^{\frac{1}{2}} z\right\|-\left\|e_{2}\right\|$

$\Rightarrow \bar{\sigma}\left(X^{\frac{1}{2}}\right)\left\|e_{1}\right\| \geq\left\|X^{\frac{1}{2}} e_{1}\right\| \geq(1-\sqrt{\alpha}) \underline{\sigma}\left(X^{\frac{1}{2}}\right)\|z\|-\left\|e_{2}\right\|(46)$

This shows that $k_{1}\left\|e_{1}\right\|+\left\|e_{2}\right\| \geq k_{2}\|z\|$, where $k_{1}, k_{2}$ are positive constants which do not depend on $\Delta$. This implies uniform robust stability.

\section{Acknowledgements}

The authors would like to thank Raff D'Andrea and A. Megretsky for numerous helpful discussions. We acknowledge AFOSR and NSF as funders of this work.

\section{References}

[1] Bercovici H., Foias C., Tannenbaum A., Structured Interpolation Theory, Op. Th. Adv. and App., 47, pp:195-220, 1990.

[2] Boyd, S.P., L El Ghaoui, E. Feron, and V. Balakrishnan, Lin. ear Matrix Inequalities in System and Contro! Theory, To be published by SIAM, April 1994.

[3] D'Andrea R., Paganini F., Doyle J.C., Uncertain Behavior, Proceedings 1993 CDC, San Antonio, Texas, pp. 3891-3896.

[4] D'Andrea R., Paganini F., Interconnection of Uncertain Behavioral Systems for Robust Control, Proceedings 1993 CDC, San Antonio, Texas, pp. 3642-3647.

[5] D'Andrea R., $\mathcal{H}_{\infty}$ Optimal Interconnections and System Design Proc. 1994 CDC, Orlando.

[6] Doyle, J., "Analysis of feedback systems with structured uncertainty" ,IEE Proceedings, 129, 242-250

[7] Doyle J., Newlin M., Paganini F., Tierno J., Unifying Robustness Analysis and System ID Proc. 1994 CDC, Orlando.

[8] Khammash M., Pearson J.B., Performance Robustness of Discrete-Time Systems with Structured Uncertainty, IEEE Trans A.C., vol AC-36, 4, pp.398-412, 1991.

[9] Lu W.M., Paganini F., Stabilization of Uncertain Linear Systems over LFT Algebras, submitted to $1995 \mathrm{ACC}$.

[10] Megretski A., Treil S., S-procedure and power distribution inequalities: $A$ new method in optimization and robustness of uncertain systems, Mittag-Leffler Inst., Rep. No 1, 1990/91.

[11] Packard A., Doyle J.C., The Complex Structured Singular Value, Automatica, Vol. 29, No. 1, pp. 71-109, 1993.

[12] Paganini F., Doyle J.C., Analysis of Implicit Uncertain Systems, in preparation.

[13] Paganini F., D'Andrea R., and Doyle J., Behavioral A pproach to Robustness Analysis Proc. 1994 ACC, Baltimore, MD.

[14] Shamma, J., Robust Stability with Time Varying Structured Uncertainty, IEEE Trans. A. C., Vol 39, 4, pp 714-724, 1994.

[15] Tierno J., Doyle J.C., Finite Time Horizon Robust Performance Analysis, Proc 1994 IEEE CDC, Orlando, Florida.

[16] Willems J.C., Paradigms and Puzzles in the Theory of Dynamical System , IEEE Trans.A.C., V.36, pp. 259-294, 1991.

[17] Young P., Robustness with Parametric and Dynamic Uncertainty $\mathrm{PhD}$ thesis, California Institute of Technology, 1993. 